\title{
Frequency-shift based detection of BMS contrast agents using SSFP: potential for MRA
}

\author{
Jessica Dubois ${ }^{\mathrm{a}}$, Franck Lethimonnier ${ }^{\mathrm{a}}$, Francoise Vaufrey ${ }^{\mathrm{a}}$, Philippe Robert ${ }^{\mathrm{b}}$, Denis Le Bihan ${ }^{\mathrm{a}, *}$ \\ ${ }^{\mathrm{a}}$ Service Hospitalier Frederic Joliot, Commissariat a l'Energie Atomique, 91401 Orsay Cedex, France \\ ${ }^{\mathrm{b}}$ Guerbet Research, 93600 Aulnay-sous-Bois, France \\ Received 31 January 2004; accepted 8 December 2004
}

\begin{abstract}
A novel mechanism of MRI contrast enhancement, based on the detection by a balanced steady-state free precession (SSFP) sequence of the proton resonance frequency shift induced by bulk magnetic susceptibility (BMS) contrast agents, was investigated. The potential for this contrast mechanism to image blood vessels was explored. The relaxation time and the frequency shift effects of gadolinium- and dysprosium-DOTA on SSFP signal was first simulated and evaluated on a water phantom at $1.5 \mathrm{~T}$. In vitro, a 5-mM concentration in contrast agent induced a $20-\mathrm{Hz}$ frequency shift, leading to a signal increase of $92 \%$ for Dy-DOTA, and a $10-\mathrm{Hz}$ frequency shift, leading to a signal increase of $58 \%$ for Gd-DOTA at the reference frequency, taking into account the nonlinear SSFP signal response on frequency offset. The concept was then evaluated in vivo on anesthetized rabbits. Low doses of dysprosium-DOTA were injected in their vascular system, and imaging was performed at the level of neck vessels. Following a bolus injection, mean signal changes of $31 \%$, 20\% and $14 \%$ were observed in the carotid arteries, the vertebral veins and the jugular veins, respectively. The bolus peak times in arteries and veins were consistent with the rabbit vascular circulation. This frequency-shift based contrast mechanism presents interesting potential for contrastenhanced MR angiography (CE-MRA) compared to usual relaxation-based contrast, but further investigations on reproducibility will be necessary.

(C) 2005 Elsevier Inc. All rights reserved.
\end{abstract}

Keywords: Contrast mechanism; Balanced SSFP; BMS contrast agents; Dysprosium chelates; MR angiography

\section{Introduction}

Steady-state free precession (SSFP) techniques, such as TrueFISP or FIESTA, are becoming widely used, as they enable high contrast-to-noise ratios to be obtained with very short acquisition times [1]. Although these techniques have been developed long ago [2-4], recent advances in fast gradient hardware have made possible their successful application in the field of cardiac MRI [5-7]. Their potential for 3-D time-of-flight (TOF) MR angiography (MRA) [8], as well as for the detection of $T_{1}$ paramagnetic contrast agents $[9,10]$, has also been reported recently. Compared to other MR imaging techniques, SSFP sequences are very sensitive to off-resonance effects. This sensitivity makes SSFP sequences suitable to detect locally induced

* Corresponding author. Tel.: +331698678 26; fax: +33169867786. E-mail address: lebihan@shfj.cea.fr (D.L. Bihan). frequency shifts. For instance, SSFP has been proposed in the context of BOLD fMRI to monitor frequency shifts induced in small vessels by their endogenous deoxyhemoglobin content [11-13].

With conventional contrast-enhanced MRA (CE-MRA), contrast enhancement of the vasculature is obtained by artificially shortening the $T_{1}$ of blood through the injection of a specific paramagnetic contrast agent, usually gadolinium chelates [14-17]. The high contrast between tissues and blood is generated by using a strongly $\mathrm{T}_{1}$-weighted radiofrequency (RF) spoiled gradient echo sequence that damps the signal of the surrounding tissue. However, relaxationbased CE-MRA presents some limitations. For instance, saturation effects may appear with high concentrations or repeated injections leading to decreased contrast and to nonlinearities in the signal-concentration relationship.

On the other hand, most contrast agents also behave as bulk magnetic susceptibility (BMS) agents, inducing a shift in the proton resonance frequency within blood vessels. Our 
aim was thus to investigate a novel mechanism of contrast enhancement in MRI using SSFP techniques to detect the frequency shift induced by BMS contrast agents. The effects of gadolinium- and dysprosium-DOTA on the SSFP signal were evaluated through theoretical simulations and in vitro experiments. The feasibility of using this contrast mechanism to detect vessels was then investigated in vivo by injection of Dy-DOTA in the vascular system of anesthetized rabbits. A preliminary account of this study was reported in Refs. [18] and [19].

\section{Theory}

\subsection{Frequency-sensitive balanced SSFP sequences}

With short TR gradient echo techniques, the MR signal $M^{x y}$ in a voxel at the time of the echo (TE) reaches a coherent steady-state level that depends on the tissue proton density $\rho$, the $T_{1}$ and $T_{2}$, the acquisition parameters (flip angle $\alpha$ and TR), and on the resonance offset angle $\beta$ acquired by the magnetization between two consecutive RF pulses [20-24]:

$\frac{M_{S S P P}^{x y}}{\rho}=\frac{\left(1-E_{1}\right)}{d} \cdot \sin \alpha \cdot e^{-T E / T_{2}} \cdot\left(1+E_{2}^{2}-2 E_{2} \cos \beta\right)^{1 / 2}$

with

$$
\begin{aligned}
& E_{i}=e^{-T R / T_{i}} \quad i=1,2 \\
& d=\left(1-E_{1} \cos \alpha\right) \cdot\left(1-E_{2} \cos \beta\right)-E_{2} \cdot\left(E_{1}-\cos \alpha\right) \cdot\left(E_{2}-\cos \beta\right) .
\end{aligned}
$$

Details on the calculation of this equilibrium signal are given in Appendix A. The resonance offset angle is given by

$\beta=\gamma \cdot \Delta B \cdot T R+\gamma \cdot \vec{r} \cdot \int_{0}^{T R} \vec{G}(t) d t+\phi_{r f}+\phi_{v}$

where $\gamma$ is the magnetogyric ratio, $\Delta B$ the local magnetic field offset, $\vec{r}$ the voxel spatial position, $\vec{G}(t)$ the spatial encoding gradients, $\phi_{\mathrm{RF}}$ the RF phase, and $\phi_{v}$ the phase contribution due to spin motion. In a perfectly balanced SSFP sequence, $\beta$, also known as the "free precession angle," is proportional to the sum of the local resonance frequency offset of the sample $v$ and of the RF receiver frequency $v_{R F}$ :

$\beta=2 \pi \cdot\left(v+v_{R F}\right) \cdot T R$

Consequently, the signal is strongly sensitive to local resonance frequency offsets: the image of a homogeneous object placed in an imperfectly shimmed field displays local signal variations. By taking advantage of this offresonance sensitivity, local shifts in proton resonance frequency $\delta v$ can be detected through variations in signal intensity (Fig. 1A). Signal increase or decrease can be selectively obtained by choosing the initial receiver

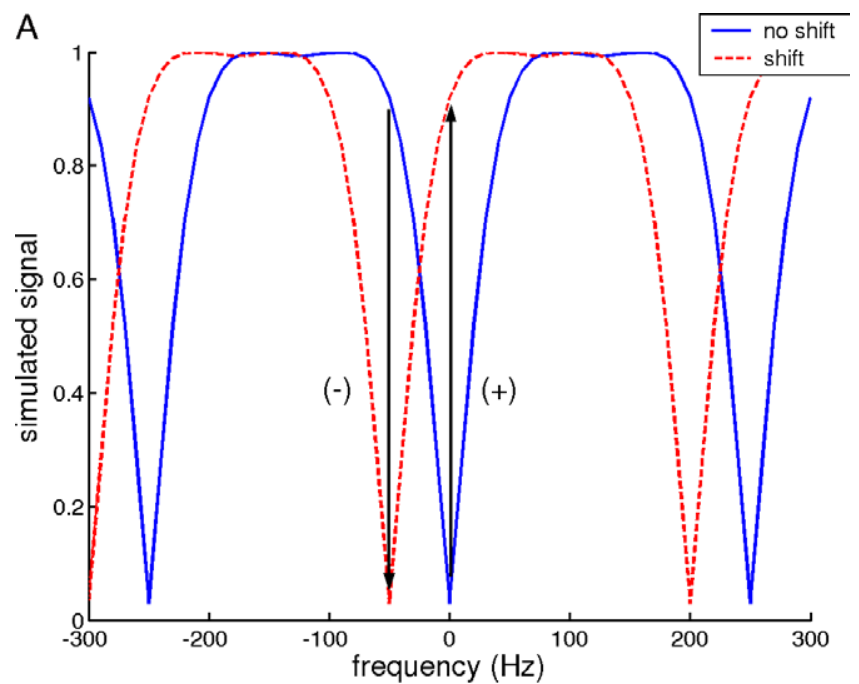

B

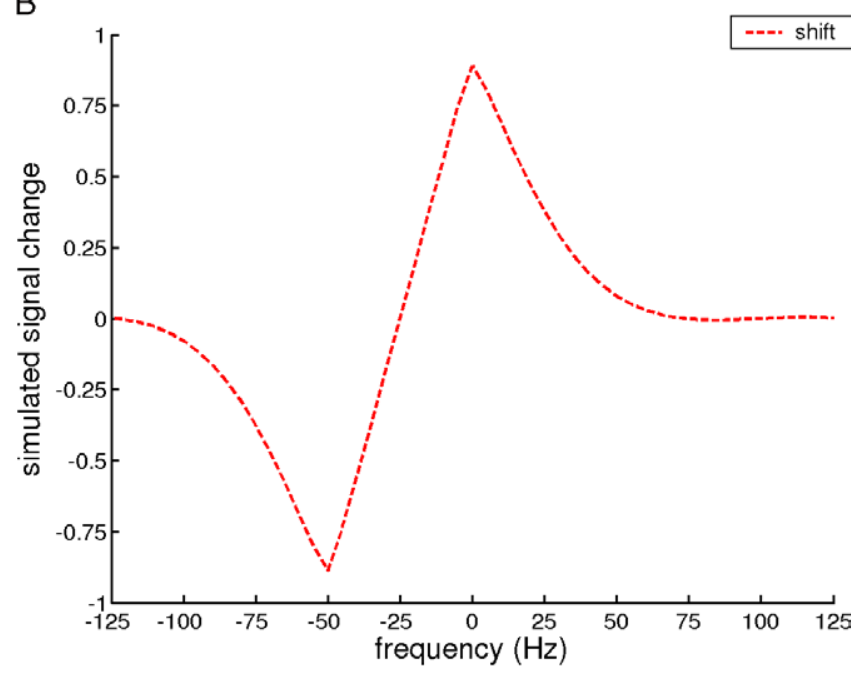

Fig. 1. Examples of simulated plots of signal versus frequency, without or with a frequency shift (A), and of signal change caused by the shift (B, obtained by subtraction of the two plots in A). According to the receiver frequency and the initial signal level, an increase $(+)$, a decrease $(-)$, or no change in signal intensity can be theoretically detected. The signal change is linear only for a short range of frequencies.

frequency (Fig. 1B). The signal change is large if the frequency is settled exactly at the minimum of the response curve, which requires the $B_{0}$ field to be shimmed uniformly to yield the most uniform nulling of the background signal in the region-of-interest.

One may object that with a change of the resonance frequency offset, the magnetization does not repeat exactly and the steady-state condition is broken. Indeed, if the phase increment "is slow compared to TR" (in other words, if the frequency increment $\delta v$ in a TR interval is small compared to $1 / \mathrm{TR}$ ), the magnetization remains in a steady state (see Ref. [25] for details in the case of a slow variation of the flip angle). Therefore, in our approach, we have assumed that the frequency shift did not affect the 
Table 1

Simulation parameters

\begin{tabular}{|c|c|c|c|c|c|c|c|}
\hline \multirow{4}{*}{$\begin{array}{l}\text { Contrast agent } \\
\text { Gd-DOTA } \\
\text { Dy-DOTA }\end{array}$} & \multicolumn{4}{|l|}{ Water } & \multicolumn{3}{|l|}{ Blood } \\
\hline & $R_{1}\left(\mathrm{~s}^{-1} \mathrm{mM}^{-1}\right)$ & $r_{2}\left(\mathrm{~s}^{-1} \mathrm{mM}^{-1}\right)$ & $\chi_{m}(\mathrm{cr}$ & $\left.\mathrm{ool}^{-1}\right)$ & $r_{1}\left(\mathrm{~s}^{-1} \mathrm{mM}^{-1}\right)$ & $r_{2}\left(\mathrm{~s}^{-1} \mathrm{mM}^{-1}\right)$ & $\chi_{m}\left(\mathrm{~cm}^{3} \mathrm{~mol}^{-1}\right)$ \\
\hline & 3.5 & 4.5 & 0.027 & & 3.85 & 4.95 & 0.027 \\
\hline & 0.11 & 0.12 & 0.048 & & 0.121 & 0.132 & 0.048 \\
\hline \multirow[t]{4}{*}{ Without contrast agent or $r>R$} & $T_{1}(\mathrm{~ms})$ & $T_{2}(\mathrm{~ms})$ & & & $T_{1}(\mathrm{~ms})$ & $T_{2}(\mathrm{~ms})$ & \\
\hline & 2600 & 1370 & & & 1200 & & \\
\hline & $T_{1}^{\prime}(\mathrm{ms})$ & $T_{2}^{\prime}(\mathrm{ms})$ & \multicolumn{2}{|c|}{$\delta v(\mathrm{~Hz}), \theta=\pi / 2$} & $T_{1}^{\prime}(\mathrm{ms})$ & $T_{2}^{\prime}(\mathrm{ms})$ & $\delta v(\mathrm{~Hz}), \theta=0$ \\
\hline & $r<R$ & $r<R$ & $r<R$ & $r>R$ & $\mathrm{r}<R$ & $\mathrm{r}<R$ & $\mathrm{r}<R$ \\
\hline$C=5 \mathrm{mM}, \mathrm{Gd}-\mathrm{DOTA}$ & 56 & 43 & -18 & 14 & 50 & 32 & 36 \\
\hline$C=5 \mathrm{mM}$, Dy-DOTA & 1070 & 752 & -32 & 24 & 695 & 136 & 64 \\
\hline$C=10 \mathrm{mM}$, Gd-DOTA & 28 & 22 & -36 & 27 & 25 & 18 & 72 \\
\hline$C=10 \mathrm{mM}$, Dy-DOTA & 674 & 518 & -64 & 48 & 489 & 125 & 128 \\
\hline
\end{tabular}

Contrast agents data were obtained from Refs. [32,33] and [35]. $T_{1}^{\prime}, T_{2}^{\prime}$, and $\delta v$ were calculated according to Eqs. (4-7). For the water simulation, both the inside compartment $(r<R)$ and the surrounding compartment $(r>R)$ were considered. Before the contrast agent is uptaken in this latter compartment, it has no effect on $T_{1}$ and $T_{2}$, but induces a frequency shift (calculated here for $r=2 R$ and $\phi=0$ ). For the blood simulation, the contrast agent has no effect on the surrounding compartment since $\theta=0$.

steady-state condition, so that the signal is simply shifted in frequency.

\subsection{Resonance frequency shift induced by BMS contrast agents}

Paramagnetic compounds have been widely used in preclinical and clinical MR studies to increase image contrast. They induce local changes in water relaxation rate constants and/or shifts in proton resonance frequency, mainly by hyperfine dipolar interactions with water hydrogen protons and BMS effects [26-29]. Bulk magnetic susceptibility effects result from the compartmentalization of the contrast agent, for instance, in blood vessels. The $T_{1}$ and $T_{2}$ modulation and the local susceptibility difference $(\delta \chi)$ between the agent compartment and surrounding tissue can be approximated by

$\frac{1}{T_{i}^{\prime}}=\frac{1}{T_{i}}+r_{i} \cdot C \quad i=1,2$

$\delta \chi=4 \pi \cdot \chi_{m} \cdot C$

where $r_{1}$ and $r_{2}$ are the longitudinal and transverse relaxivities of the contrast agent, $C$ the agent concentration, and $\chi_{\mathrm{m}}$ the agent molar susceptibility. In general, metal chelation can dramatically affect relaxivities but has a much smaller effect on susceptibility.

The water resonance frequency shift $\delta \nu$ induced by a BMS contrast agent in the vascular compartment depends on the shape of the vessel and on its orientation relative to $B_{0}$. By modeling a vessel by an infinite cylinder and correcting for the sphere of Lorentz, a construct employed to account for molecular scale susceptibility cancellation, this frequency shift, $\delta \nu$, is

$$
\delta v=\frac{\gamma B_{0}}{4 \pi} \cdot \delta \chi \cdot\left(\cos ^{2} \theta-\frac{1}{3}\right)
$$

where $\theta$ is the angle between $B_{0}$ and the cylinder axis [29-31]. In the surrounding compartment, the frequency shift $\delta v{ }^{\text {ext }}$ is given by

$$
\delta v^{\mathrm{ext}}(r, \phi)=\frac{\gamma B_{0}}{4 \pi} \cdot \delta \chi \cdot \sin ^{2} \theta \cdot \cos (2 \phi) \cdot\left(\frac{R}{r}\right)^{2}, \text { for } r>R
$$

where $R$ is the cylinder radius, $r$ the distance to the cylinder axis, and $\phi$ the polar angle between $r$ and the component of $B_{0}$ in the plane normal to the cylinder. If the cylinder axis is parallel to $B_{0}(\theta=0)$, there is no frequency shift.

Elements of the lanthanide series exhibit different magnetic properties [26-28,32,33]. Gadolinium is both an efficient dipolar relaxation enhancer and a susceptibility agent. On the contrary, dysprosium is mainly an efficient susceptibility agent, inducing larger frequency shifts (more than $50 \mathrm{~Hz}$ at $5 \mathrm{mM}$ and $1.5 \mathrm{~T}$ ).

With a balanced SSFP technique, a strong signal difference should thus be observed between BMS contrast agent filled vessels and surrounding tissues because of the frequency shift induced. In this study, this contrast mechanism was evaluated with gadolinium and dysprosium chelates.

\section{Methods}

\subsection{Simulations}

The effects of contrast agents on SSFP signal intensity was first evaluated through simulations with Matlab 5.3 Software (MathWorks, Natick, MA). The agents were assumed to be in a water compartment perpendicular to $B_{0}(\theta=\pi / 2)$ and in a blood compartment parallel to $B_{0}$ $(\theta=0)$, to model, respectively, the phantom for the in vitro experiments and the rabbit neck vessels for the in vivo 
experiments. For the outside compartment $(r>R)$, the simulation was considered only in the perpendicular case, as the frequency shift cancels out when $\theta=0$. Both relaxation time effects and resonance frequency shifts at $1.5 \mathrm{~T}$ were taken into account. Signal variations induced by contrast agents were evaluated for the frequency which minimizes signal in the absence of contrast agent. Contrast enhancement is presented in percentage of the maximum of theoretical signal in water (noted $S_{0}^{\text {th }}$ ).

\subsection{MRI data acquisition}

Experiments were performed on phantoms and on anesthetized rabbits. The FIESTA sequence was implemented in a 1.5-T MRI system (Signa LX, GEMS). For the
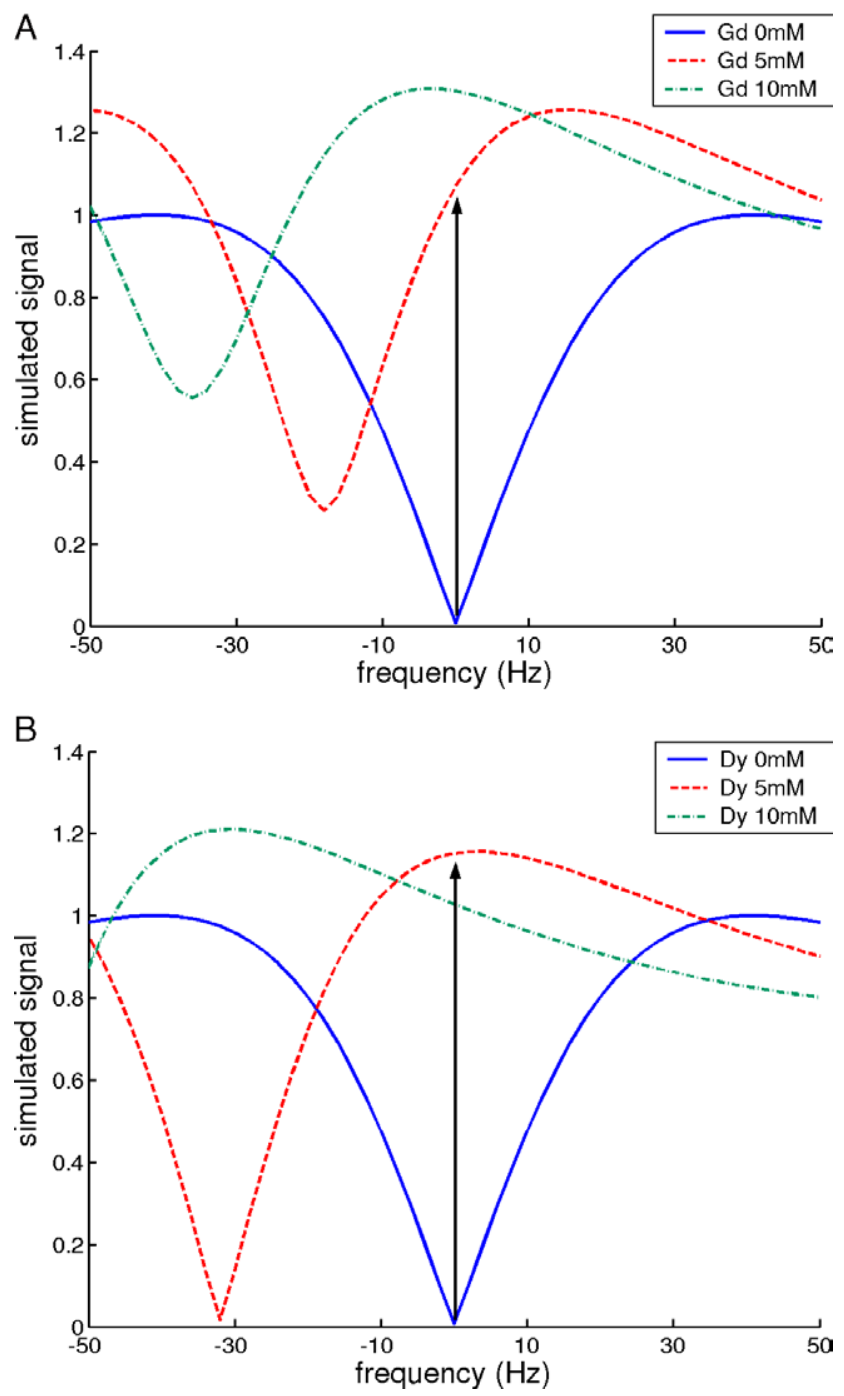

Fig. 2. Simulated plots of signal versus frequency for a $3.5-\mathrm{ms}$ TR and a $35^{\circ}$ flip angle, in the water compartment with $\theta=\pi / 2$, for gadolinium (A) and dysprosium (B) at different concentrations $(0,5,10 \mathrm{mM})$. The signal variations described in the text were obtained for the frequency which minimized signal in the absence of contrast agent $(v=0$, arrows in the figures).
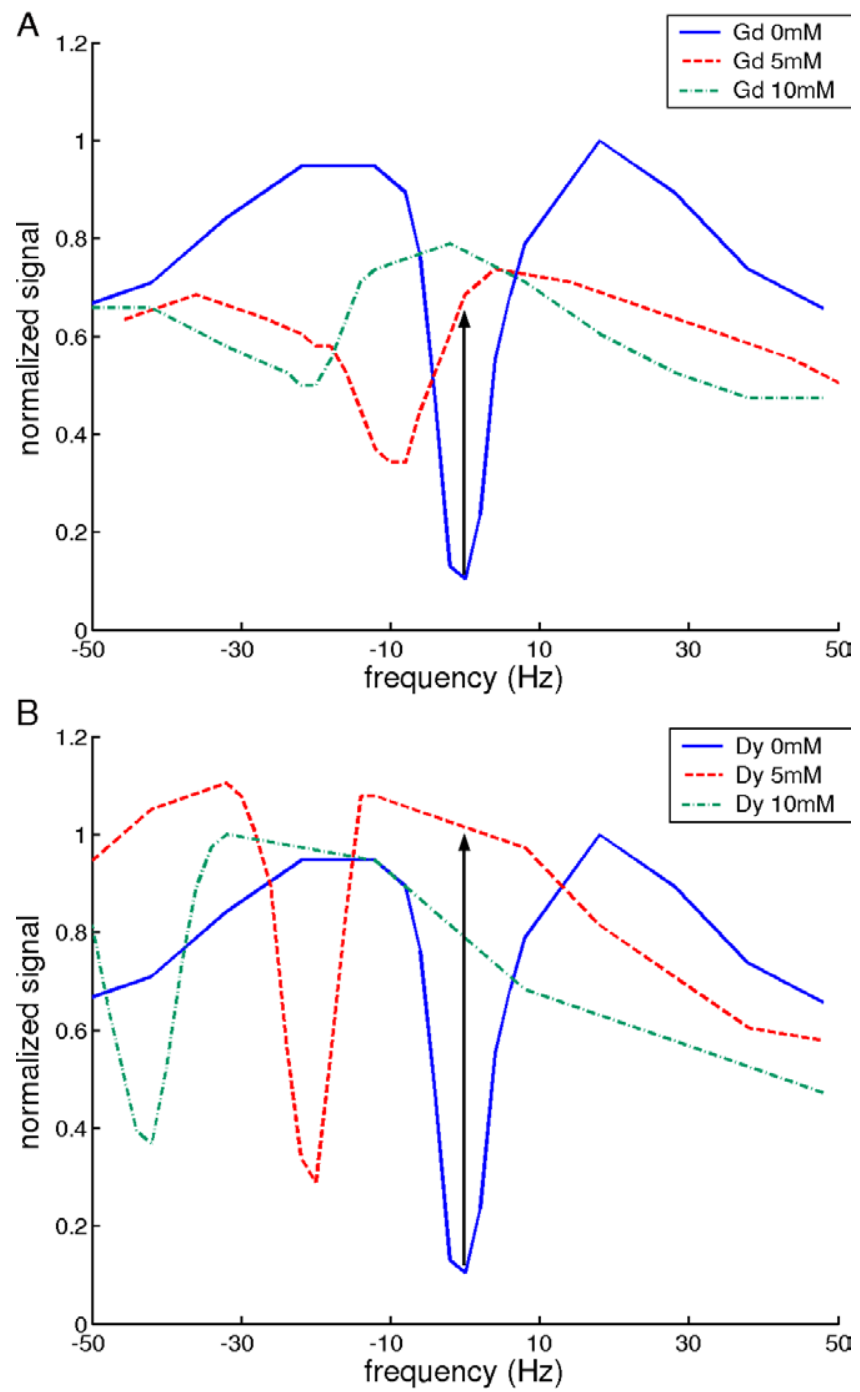

Fig. 3. In vitro experimental plots of normalized signal versus frequency for solutions of Gd-DOTA (A) and Dy-DOTA (B) at different concentrations $(0,5,10 \mathrm{mM})$. The signal variations described in the text were obtained for the frequency which minimized signal in the absence of contrast agent $(v=0$, arrows in the figures).

in vitro experiments, a head coil was used. The acquisition parameters were $20-\mathrm{cm}$ FOV, $128 \times 128$ matrix, $1.56 \times$ $1.56 \times 3-\mathrm{mm}^{3}$ voxels, $35^{\circ}$ flip angle, $125-\mathrm{kHz}$ bandwidth, 3.5-ms TR, $1.7-\mathrm{ms} \mathrm{TE}$, and 100 preparation RF pulses. A coronal slice was acquired in $1.4 \mathrm{~s}$. For the in vivo experiments, a GP-FLEX surface coil was used. The parameters were $12-\mathrm{cm}$ FOV, $96 \times 96$ matrix, $1.25 \times$ $1.25 \times 4-\mathrm{mm}^{3}$ voxels, $35^{\circ}$ flip angle, $62.5-\mathrm{kHz}$ bandwidth, 4.1-ms TR, 1.9-ms TE, and 30 preparation RF pulses. A $35^{\circ}$ flip angle was used because it theoretically maximizes the on-resonance SSFP signal in blood [20]. An axial slice was acquired in $850 \mathrm{~ms}$, at the rabbit neck level. Time-of-flight and phase-contrast acquisitions were also performed in order to precisely localize the vessels and provide a basis for comparison with the SSFP approach. 


\subsection{In vitro experiments}

Experimental evaluation was first performed on a phantom roughly modeling vessel geometry (a cylindrical tube with a ratio between length and diameter of $6: 1$ ). The tube, perpendicular to $B_{0}$ and surrounded by water, was filled with various solutions of extracellular contrast agents, Gd-DOTA and Dy-DOTA (Guerbet Research, Aulnay-sousBois, France), at different concentrations (0, 5, $10 \mathrm{mM})$. The concentration range matched those expected in a blood vessel following a bolus injection (the arterial blood agent peak concentration is typically of $10 \mathrm{mM}$ for a clinical dose of $0.1 \mathrm{mmol} \mathrm{kg}{ }^{-1}$ [34]). In order to assess the relaxation effects and the frequency shift amplitudes induced by the contrast agents, images were acquired with several acquisition RF pulse frequency offsets, over a range of $100 \mathrm{~Hz}$, with a $2-\mathrm{Hz}$ step. The signal was measured in a region-ofinterest of 70 voxels in the tube.

The signal intensities in water and in the contrast agent solutions were compared for the acquisition frequency which minimized the signal in water. In Results, contrast enhancement is presented in percentage of the maximum of experimental signal in water (noted $S_{0}^{\exp }$ ).

\subsection{In vivo experiments}

Feasibility for MRA was then evaluated on three mature New Zealand white rabbits (mean weight, $2.5 \mathrm{~kg}$ ). The experiments were performed in accordance with all institutional regulations governing the performance of animal studies. During the experiments, the rabbits were under full anesthesia with ketamine (Imalgene 1000: $0.5 \mathrm{ml} \mathrm{kg}^{-1}$ ) and xylazine (Rompun $2 \%: 0.5 \mathrm{ml} \mathrm{kg}^{-1}$ ). The neck vessels were approximately parallel to $B_{0}$.

Before each injection, images were first acquired by scanning different RF pulse frequencies, to pick-up the frequency minimizing the signal in most of the slice. One test was also purposely performed at a nonoptimal frequency to bear out frequency shift effects and reveal residual relaxation effects, if present.

To inject the contrast agent in the vascular system of the rabbit, the ear marginal vein was catheterized (Insyte Vialon
$22 \mathrm{G})$. Based on the in vitro experiment results, only Dy-DOTA was used. Bolus injections were performed (0.1-mmol kg-1 dose) and repeated (two injections per rabbit, separated by $10 \mathrm{~min}$ ). The agent was injected manually, using a 5-ml syringe connected to the catheter. The volume was adjusted to $2.5 \mathrm{ml}$ with saline before injection. The mean injection rate was $0.3 \mathrm{ml} \mathrm{s}^{-1}$.

With the selected acquisition frequency, 400 images were acquired before, during, and after the injection. The signal time course was measured in regions-of-interest of seven voxels in the carotid arteries and the vertebral and jugular veins. Signal was normalized by maximal signal obtained in a water phantom $\left(S_{0}^{\text {exp }}\right)$.

The FIESTA signal stability was assessed before injection in the rabbit vessels over 200 baseline images. It is presented in percentage in Results (standard deviation divided by mean intensity). To evaluate the effect of Dy-DOTA injection on signal, contrast enhancement was calculated by comparing the peak and the mean baseline intensities (in percentage of $\left.S_{0}^{\exp }\right)$. Peak times of agent detection were recorded in vessels from the end of the injection. Finally, image subtraction was performed to selectively visualize vessels at different time points. A mean image of the neck before injection was calculated to smooth out flow effects. This image was then subtracted from the images acquired at peak signal changes in the vessels.

\section{Results}

\subsection{Simulations}

Simulation parameters (relaxation times and resonance frequency shifts) are detailed for gadolinium and dysprosium at a 0-, 5-, 10-mM concentration in Table 1. Plots of theoretical signal in the water compartment versus frequency are presented in Fig. 2. For gadolinium (Fig. 2A) at $5 \mathrm{mM}$, a decrease in $T_{1}$ (from 2600 to $56 \mathrm{~ms}$ ) and $T_{2}$ (from 1370 to $43 \mathrm{~ms}$ ), leading to an increase in maximal and minimal signal, respectively, of $26 \%$ and $27 \%$ of $S_{0}^{\text {th }}$, and a $18-\mathrm{Hz}$ frequency shift were predicted. Both relaxation time effects and frequency shift induced an important SSFP

Table 2

Signal changes and peak times following in vivo bolus injections

\begin{tabular}{|c|c|c|c|c|c|c|}
\hline \multirow[t]{2}{*}{ Injection number } & \multicolumn{3}{|c|}{ Signal variation $\left(\% S_{0}^{\exp }\right)$} & \multicolumn{3}{|l|}{ Peak time (s) } \\
\hline & Jugular vein & Carotids & Vertebral veins & Jugular vein & Carotids & Vertebral veins \\
\hline 1 & -7 & 21 & 9 & 0 & 4.1 & 5.4 \\
\hline 2 & -7 & 15 & -23 & 2.7 & 8.1 & 8.6 \\
\hline 3 & -38 & -56 & -24 & 0.9 & 4.3 & 5.1 \\
\hline 4 & - & - & _- & - & _ & - \\
\hline 5 & -3 & -35 & 16 & 0 & 4.3 & 7.7 \\
\hline 6 & 16 & -28 & 30 & 5.6 & 6.3 & 15.4 \\
\hline Mean & 14 & 31 & 20 & 1.8 & 5.4 & 8.4 \\
\hline S.D. & 14 & 16 & 8 & 2.4 & 1.7 & 4.2 \\
\hline
\end{tabular}

Injections 1 and 2 were performed in the first rabbit, injections 3 and 4 in the second one, and injections 5 and 6 in the third one. No signal change was observed following injection 4 , which was purposely performed at a nonoptimal acquisition frequency. Means and standard deviations were calculated according to absolute values. 
signal variation for the frequency which minimized signal in the absence of contrast agent $\left(95 \%\right.$ of $S_{0}^{\text {th }}$ for $\left.v=0\right)$. In comparison, dysprosium at $5 \mathrm{mM}$ induced a significant frequency shift $(32 \mathrm{~Hz})$ and reduced decrease in $T_{1}$ (from 2600 to $1070 \mathrm{~ms}$ ) and $T_{2}$ (from 1370 to $752 \mathrm{~ms}$ ), which led to an increase in maximal and minimal signal, respectively, of $16 \%$ and $1 \%$ of $S_{0}^{\text {th }}$ (Fig. 2B). This also led to a significant signal change for the frequency which minimized signal in the absence of contrast agent $(115 \%$ of $S_{0}^{\text {th }}$ for $\left.v=0\right)$, but through a different mechanism.

\subsection{In vitro experiments}

Experimental plots of normalized signal (compared to $S_{0}^{\exp }$ ) versus frequency are presented in Fig. 3. Signal in water differed from simulation on two points. First, the minimum signal was not zero but $10 \%$ of $S_{0}^{\exp }$ (noise level). Second, the range of frequencies inducing a fall in signal intensity was shallower than expected (full-width half maximum of $8 \mathrm{~Hz}$ instead of $22 \mathrm{~Hz}$ ).

The effects of contrast agents on signal were compared. With respect to water, Gd-DOTA induced a frequency shift of $10 \mathrm{~Hz}$ at $5 \mathrm{mM}$ and important $T_{1}$ and $T_{2}$ effects (decrease in maximal signal of $26 \%$ of $S_{0}^{\exp }$ and increase in minimal signal of $24 \%$ of $S_{0}^{\exp }$ ) (Fig. 3A). A $5-\mathrm{mM}$ concentration increased signal by $58 \%$ of $S_{0}^{\exp }$, at the reference frequency (minimizing the signal in the absence of contrast agent, $v=0$ ). As expected, Dy-DOTA induced a more important frequency shift $(20 \mathrm{~Hz}$ at $5 \mathrm{mM})$, and the relaxation time effects were lower (increase in maximal and minimal signal, respectively, of $10 \%$ and $18 \%$ of $S_{0}^{\exp }$ ) (Fig. 3B). The increase in signal intensity was $92 \%$ of $S_{0}^{\exp }$ for a $5-\mathrm{mM}$ concentration, at the reference $(v=0)$.

Although the experimental signal change was smaller than predicted by simulation, the frequency shift effect on signal was more important than relaxation effects. Hence, the signal change induced by Dy-DOTA was almost twice that induced by Gd-DOTA at identical concentration.

\subsection{In vivo experiments}

Before contrast agent injection, fluctuations in the signal time course amplitude of about $27 \%, 12 \%$, and $7 \%$ were observed in the carotids, the vertebral veins, and the jugular veins, respectively, because of the pulsating nature of the flow $[36,37]$. In surrounding tissues, signal stability was within $4 \%$. Due to imperfect shimming, it was not possible to find a unique acquisition frequency minimizing signal throughout the entire slice. Therefore, the frequency was optimized for the slice center.

Following the bolus injections, Dy-DOTA-filled vessels were clearly visible through induced signal variations (Table 2). Examples of normalized signal time courses are presented in Fig. 4 for three different injections (numbers 1, 3, and 5 in Table 2). In a vessel, signal level was either increased or decreased, according to the local vessel baseline frequency (see Fig. 1B). Mean changes in intensity of $31 \%, 20 \%$, and $14 \%$ of $S_{0}^{\exp }$ were, respectively,
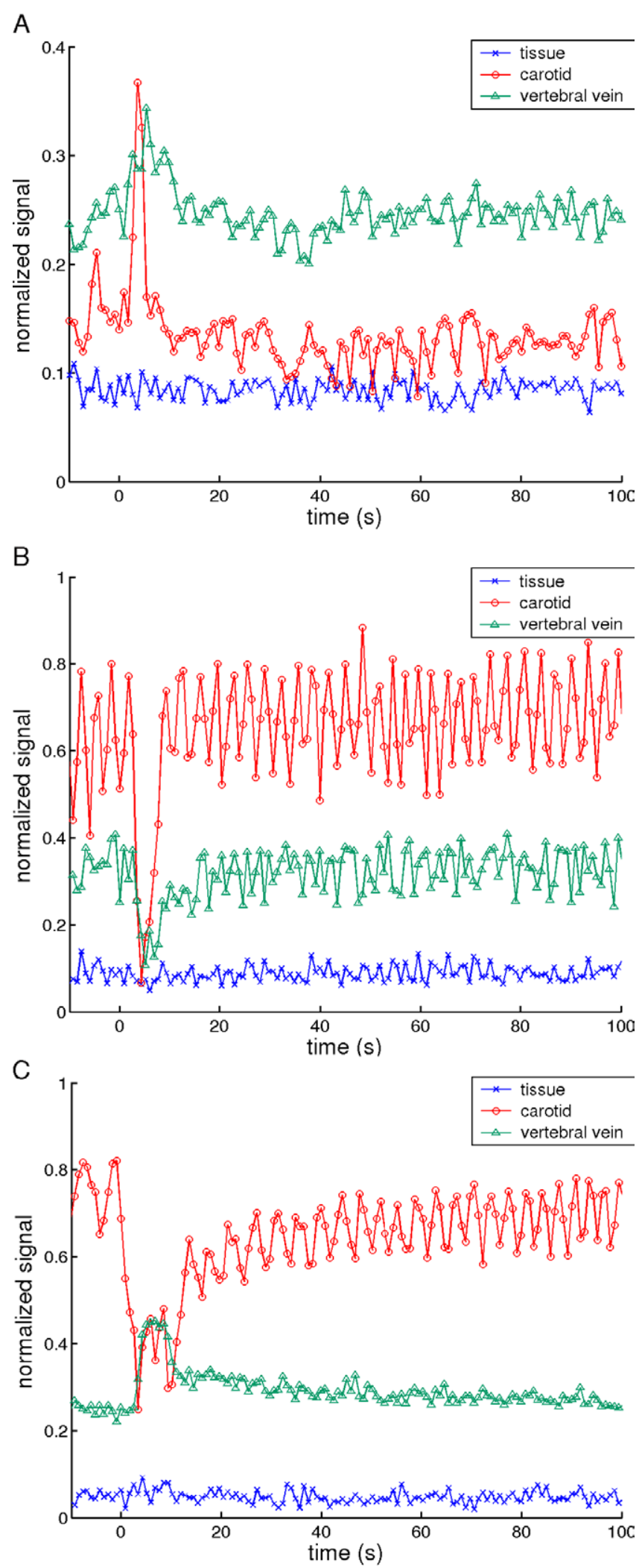

Fig. 4. Examples of in vivo normalized signal time course in a carotid, a vertebral vein, and a tissue following a Dy-DOTA bolus injection at $0 \mathrm{~s}$ time for three different injections (numbers 1,3, and 5 in Table 2). A signal increase or decrease was observed in the vessels, depending on the adjustment of the receiver frequency. 

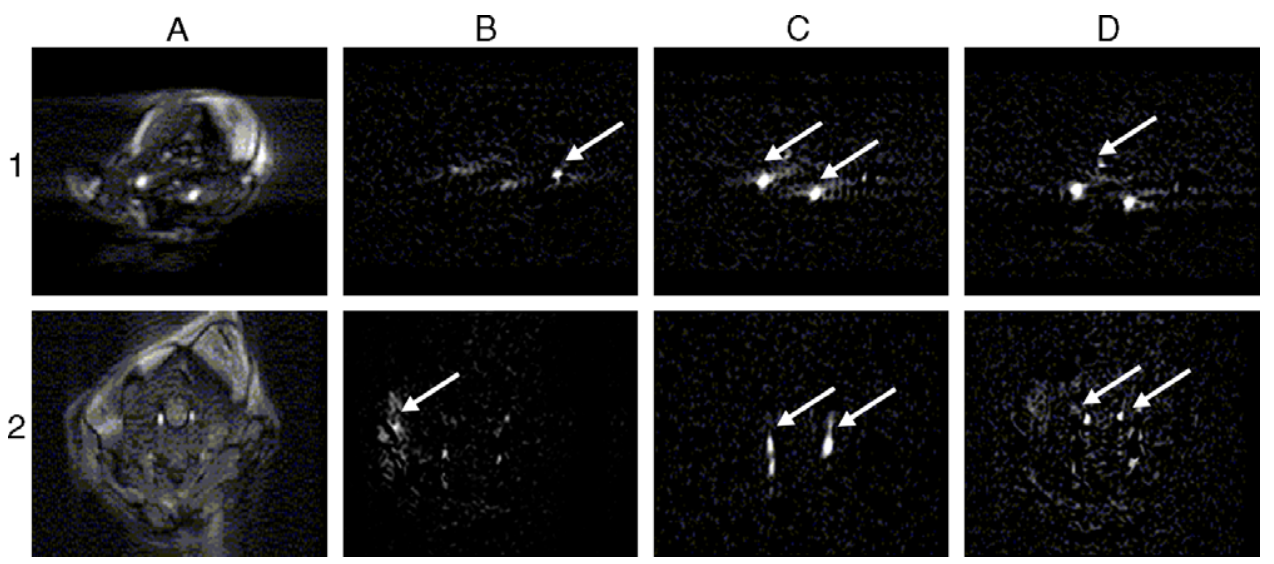

Fig. 5. Images of the vessels for two rabbits (1-2): mean image of the neck before injection (A), jugular vein on the injection side (B), carotids (C), vertebral veins (D). The vessel images were obtained by subtracting the mean image before injection and the image acquired at different time points (corresponding to signal changes peaks). According to signal time course, the obtained images correspond to carotids or veins.

observed in the carotid arteries, the vertebral veins, and the jugular vein compared to baseline. Signal peaks were observed in vessels at different times, consistently with the contrast agent vascular circulation pattern (Table 2). An early signal change was observed in average $1.8 \mathrm{~s}$ after the end of the injection in the jugular vein on the injection side, while no change was detected on the contralateral side. In the carotids and in the vertebral veins, the bolus was detected, respectively, 5.4 and $8.4 \mathrm{~s}$ in average after the end of the injection.

The experiment performed with a nonoptimal acquisition frequency (number 4 in Table 2) led to no significant signal change, within the normal signal fluctuations, in vessels after bolus injection, confirming that relaxation effects were negligible with Dy-DOTA. The possibility of repeating contrast agent injections was evaluated. With conventional contrast agents, repeated injections are prohibited since they lead to saturation effects. With Dy-DOTA agent, several injections would lead to a further frequency shift, so equivalent signal changes would be expected, providing that the receiver frequency is adjusted before each injection at the minimum of the signal curve, in order to decrease the baseline signal. Experimentally, after two successive injections, equivalent absolute signal change amplitudes were observed (Table 2).

The distinction between arteries and veins was easily obtained by subtracting the mean image before injection and images acquired at different time points, corresponding to peak signal changes in the respective vessels (Fig. 5). The vessels colocalized with those observed with the TOF and phase-contrast acquisitions.

\section{Discussion}

This work introduces a novel mechanism for contrast enhancement in MRI using balanced SSFP techniques to detect the frequency shift induced by BMS contrast agents.
The effects of gadolinium and dysprosium chelates on signal were evaluated. The feasibility of using this mechanism to detect blood vessels was explored.

Experiments on phantoms demonstrated that Dy-DOTA, a still rarely used shift reagent producing important frequency shifts, induced larger signal variations than Gd-DOTA. Relaxation and frequency shift effects could be decoupled by measuring the signal at different acquisition frequency offsets. Relaxation effects were shown to be small, while most observed signal changes were linked to the frequency shift. However, the shift observed in vitro was lower than expected from theory. A possible source for this discrepancy is that the theory assumed a truly cylindrical infinite phantom perpendicular to $B_{0}$ whereas the tube size was finite.

Experiments performed on anesthetized rabbits highlighted the potential and the limits of the contrast mechanism to image blood vessels. Dy-DOTA is an extracellular agent that is rapidly eliminated. A clinically acceptable dose was used. After bolus injections, high signal changes compared to baseline could be observed in the neck vessels. The signal changes peak could be observed at times consistent with the vascular circulation physiology. The contrast agent first pass was observed in vessels and enabled to distinguish between arteries and veins. Images of the vessels could then be produced.

The method that consists in detecting a frequency shift induced by a contrast agent presents advantages compared to the detection of relaxation effects. Using conventional, relaxivity-based agents, signal saturation effects occur, which make the use of multiple injections difficult (typically at least $15 \mathrm{~min}$ are necessary between injections with rapid-clearance contrast agents). With the proposed approach, protocols requiring repeated injections will be easier to implement as frequency shifts will add without saturation effects, providing that the receiver frequency is adjusted before each injection. Even if an uptake of the 
contrast agent in the surrounding tissues causes an additional frequency offset, which might blur the contrast between the vessel and the tissue, it is always possible to shift the signal curve versus frequency. Furthermore, the use of a dysprosium-based blood pool contrast agent could improve the CNR available between vessels and tissues.

Another approach has been suggested to image blood vessels using chemical shift contrast agents [38]. The method consists in suppressing nonshifted water signal with a binomial pulse and a spoiling gradient introduced before a 3-D gradient echo sequence. The major drawback is that it requires high dose $\left(1.2 \mathrm{mmol} \mathrm{kg}^{-1}\right)$ to induce large frequency shift $(300 \mathrm{~Hz})$. With our approach, a clinically acceptable dose of agent also led to significant effects.

With our low-dose method, the signal change amplitude was equivalent with those found in the MRA literature for relaxation contrast agents. For Gd-DOTA, Scheffler et al. [9] reported blood signal changes of about $15 \%$ to $25 \%$ of $M_{0}$ with conventional imaging methods and $40 \%$ to $50 \%$ of $M_{0}$ with $T_{1}$-weighted FIESTA imaging. Foo et al. [10] described a stable relative signal increase of $50 \%$ of maximal signal obtained in blood following an injection of $0.2 \mathrm{mmol} \mathrm{kg}^{-1}$ of gadoteridol with FIESTA.

The proposed contrast mechanism still presents several drawbacks for its application in CE-MRA. First, SSFP technique is sensitive to flow [36,37]. However, although the signal was not perfectly stable, it reached an average level that was always sufficiently high for vessel detection. Second, the observation of a signal increase or decrease was a "predictable drawback." It is intrinsically caused by the frequency shift origin of the signal enhancement. As outlined in the theoretical section, the initial signal in a voxel depends on its initial local resonance frequency offset. According to this offset, a frequency shift can induce an increase, a decrease, or no change in signal intensity (see Fig. 1B). Therefore, following contrast agent injection, the sign of signal changes should strongly depend on the acquisition frequency offset selected before injection. This dependence was experimentally observed. Furthermore, the test purposely performed at a nonoptimal frequency led to no significant signal change, as expected. This technique, thus, requires particular care in adjusting the acquisition parameters (shimming and frequency offset). Third, signal was not homogeneous throughout the slice because of underlying $B_{0}$ variation due to imperfect shimming and normal susceptibility variation in the rabbit neck. The imperfect shimming prevented us from finding a single frequency offset to minimize baseline signal in all vessels. Moreover, the variations in the form and orientation of the vessels might explain why the amplitude and sign of the signal changes following contrast agent injection were variable within the vessels and the experiments. Actually, the BMS effect should go to zero in the inside compartment for vessels making a specific angle with $B_{0}\left(\theta=\cos ^{-1}(1 / \sqrt{3})\right)$ and in the surrounding compartment for vessels parallel to $B_{0}$.
For this approach to be clinically useful, several improvements should be first considered to increase reliability. Especially, shim homogeneity should be increased over the imaged volume. Moreover, analyzing signal phase should be helpful. The phase is only discontinuous for the signal minimum $[1,39,40]$. Therefore, by adjusting the acquisition frequency before injection to obtain a single phase through the whole slice, the sign of signal variation following the frequency shift could be identical in all vessels. However, the proposed contrast mechanism might be particularly suitable for imaging small fields-of-view or regions-of-interest with higher field homogeneity, as in microcirculation and tumor imaging. Another potential application would be "molecular" imaging: as the BMS effect depends on the contrast agent compartmentalization, cells which have internalized a BMS agent would exhibit a frequency shift and could, therefore, be detected with the SSFP technique.

Another important feature of this approach is that the effect is expected to increase linearly with $B_{0}$. As "clinical" high-field MRI systems operating at $3 \mathrm{~T}$ or above are rapidly extending, shift reagents may become especially useful, as the effects of conventional relaxation-based agents decrease with increasing $B_{0}$. Nevertheless, the greater difficulty in shimming at high field should not be underestimated.

Finally, from another perspective, our study highlights the limits of using SSFP techniques for the detection of gadolinium chelates based on relaxation effects at high field. Off-resonance effects are artifacts in conventional methods, and the acquisition frequency is set to minimize them. This may not be possible with increasing field and TR, because BMS contrast agents would induce reasonable frequency shift, which would add to or cancel the relaxation effects detected with SSFP.

\section{Conclusion}

The monitoring of the proton frequency shift induced by BMS contrast agents with SSFP techniques is a novel mechanism of contrast enhancement in MRI. Strong contrast between vessels and tissue can be obtained in real time following injections of Dy-DOTA, but it requires that the $B_{0}$ field is uniformly shimmed in the region-of-interest. Because there is no saturation effect due to $T_{1}$ and $T_{2}$ relaxation, repeated bolus injections can be detected without loss of sensitivity, when the receiver frequency is adjusted before each injection. In the future, this contrast mechanism might become an alternative approach for CE-MRA, on condition that method reproducibility is improved.

\section{Acknowledgments}

The authors thank Patrick Le Roux from General Electric Medical System (Buc, France) for discussion and support on the FIESTA sequence. 


\section{Appendix A}

\section{SSFP signal}

This appendix is derived from Ref. [20] and describes the calculation of SSFP signal. The magnetization status before and after a RF pulse are noted $\vec{M}^{-}$and $\vec{M}^{+}$, respectively.

The $n$th RF pulse is first considered. The rotation through a flip angle $\alpha$ around the $x$ axis implies that

$\vec{M}^{+}(n)=R_{x}(\alpha) \vec{M}^{-}(n)$

where $R_{x}(\alpha)=\left(\begin{array}{ccc}1 & 0 & 0 \\ 0 & \cos \alpha & \sin \alpha \\ 0 & -\sin \alpha & \cos \alpha\end{array}\right)$.

During the TR time interval, the magnetization acquires a resonance offset angle $\beta$ around the $z$ axis and evolves through longitudinal $T_{1}$ recovery and transverse $T_{2}$ relaxation. Therefore, the magnetization existing before the $n$th pulse and after the $(n-1)$ th pulse is linked through:

$\vec{M}^{-}(n)=D(T R) \cdot \vec{M}^{+}(n-1)+\rho\left(1-E_{1}\right) \cdot \vec{z}$

where $\rho$ is the proton density,

$D(T R)=\left(\begin{array}{ccc}E_{2} \cos \beta & E_{2} \sin \beta & 0 \\ -E_{2} \sin \beta & E_{2} \cos \beta & 0 \\ 0 & 0 & E_{1}\end{array}\right)$

and

$E_{i}=e^{-T R / T_{i}} \quad i=1,2$.

Besides, the steady-state condition implies that

$\vec{M}_{S S F P}^{-}(n)=\vec{M}_{S S F P}^{-}(n-1)$ and

$\vec{M}_{S S F P}^{+}(n)=\vec{M}_{S S F P}^{+}(n-1)$.

By solving the equations

$\frac{\vec{M}_{S S F P}^{-}}{\rho}=\left(1-E_{1}\right) \cdot\left[1-D(T R) \cdot R_{x}(\alpha)\right]^{-1} \cdot \vec{z}$

and

$\vec{M}_{S S F P}^{+}=R_{x}(\alpha) \cdot \vec{M}_{S S F P}^{-}$,

the magnetization at equilibrium can be obtained before and after a RF pulse, respectively:

$$
\begin{aligned}
\frac{\vec{M}_{S S F P}^{-}}{\rho}= & \frac{\left(1-E_{1}\right)}{d} \\
& \times\left(\begin{array}{c}
E_{2} \sin \beta \sin \alpha \\
E_{2} \sin \alpha\left(\cos \beta-E_{2}\right) \\
\left(1-E_{2} \cos \beta\right)-E_{2} \cos \alpha\left(\cos \beta-E_{2}\right)
\end{array}\right) \\
\frac{\vec{M}_{\text {SSFP }}^{+}}{\rho}= & \frac{\left(1-E_{1}\right)}{d} \\
& \times\left(\begin{array}{c}
E_{2} \sin \beta \sin \alpha \\
\sin \alpha\left(1-E_{2} \cos \beta\right) \\
\cos \alpha\left(1-E_{2} \cos \beta\right)-E_{2}\left(\cos \beta-E_{2}\right)
\end{array}\right)
\end{aligned}
$$

where

$$
d=\left(1-E_{1} \cos \alpha\right) \cdot\left(1-E_{2} \cos \beta\right)-E_{2} \cdot\left(E_{1}-\cos \alpha\right) \cdot\left(E_{2}-\cos \beta\right) .
$$

Finally, the magnetizations in the transverse plane, respectively, after and before the RF pulse, and at the time of the echo (TE) are

$$
\begin{aligned}
& \frac{M_{S S F P}^{+x y}}{\rho}=\frac{\left(1-E_{1}\right)}{d} \cdot \sin \alpha \cdot\left(1+E_{2}^{2}-2 E_{2} \cos \beta\right)^{1 / 2} \\
& M_{S S F P}^{-x y}=E_{2} \cdot M_{S S F P}^{+x y} \\
& M_{S S F P}^{x y}=e^{-T E / T_{2}} \cdot M_{S S F P}^{+x y}
\end{aligned}
$$

\section{References}

[1] Scheffler K, Hennig J. Is TrueFISP a gradient-echo or a spin-echo sequence? Magn Reson Med 2003;49:395-7.

[2] Carr HY. Steady-state free precession in nuclear magnetic resonance. Phys Rev Lett 1958;112:1693-701.

[3] Oppelt A, Graumann R, Barfub H, Fischer H, Hartl W, Schajor W. FISP: A new fast MRI sequence (German). Electromedica 1986;54: $15-8$.

[4] Sekihara K. Steady-state magnetizations in rapid NMR imaging using small flip angles and short repetition intervals. IEEE Trans Med Imaging 1987; MI6:157-64.

[5] Plein S, Bloomer TN, Ridgway JP, Jones TR, Bainbridge GJ, Sivananthan MU. Steady-state free precession magnetic resonance imaging of the heart: Comparison with segmented k-space gradientecho imaging. J Magn Reson Imaging 2001;14:230-6.

[6] Jung BA, Hennig J, Scheffler K. Single-breathhold 3D-TrueFISP cine cardiac imaging. Magn Reson Med 2002;48:921-5.

[7] Schreiber WG, Schmitt M, Kalden P, Mohrs OK, Kreitner KF, Thelen M. Dynamic contrast-enhanced myocardial perfusion imaging using saturation-prepared TrueFISP. J Magn Reson Imaging 2002;16: $641-52$.

[8] Leupold J, Hennig J, Scheffler K. 3D Time-of-flight MRA using inversion recovery TrueFISP. Proceedings of the 10th Annual Meeting of ISMRM. Honolulu, Hawaii; 2002. p. 138.

[9] Scheffler K, Winterer J, Langer M, Hennig J. Contrast-enhanced angiography using $\mathrm{T}_{1}$-weighted TrueFISP. Proceedings of the 10th Annual Meeting of ISMRM. Honolulu, Hawaii; 2002. p. 139.

[10] Foo TKF, Ho VB, Marcos HB, Hood MN, Choyke PL. MR angiography using steady-state free precession. Magn Reson Med 2002;48:699-706.

[11] Scheffler K, Seifritz E, Bilecen D, Venkatesan R, Hennig J, Deimling $\mathrm{M}$, et al. Detection of BOLD changes by means of a frequencysensitive TrueFISP technique: Preliminary results. NMR Biomed 2001;14:490-6

[12] Dubois J, Lethimonnier F, Klein I, Poline JB, Le Bihan D. Frequencyshift based BOLD fMRI using SSFP in human visual cortex. Proceedings of the 10th Annual Meeting of ISMRM. Honolulu, Hawaii; 2002. p. 117.

[13] Miller KL, Hargreaves BA, Lee J, Ress D, Christopher DeCharms R, Pauly JM. Functional brain imaging using a blood oxygenation sensitive steady state. Magn Reson Med 2003;50:675-83.

[14] Prince MR, Yucel EK, Kaufman JA, Harrison DC, Geller SC. Dynamic gadolinium-enhanced three-dimensional abdominal MR arteriography. J Magn Reson Imaging 1993;3:877-81.

[15] Prince MR. Gadolinium-enhanced MR aortography. Radiology 1994;191:155-64. 
[16] Snidow JJ, Johnson MS, Harris VJ, Margosian PM, Aisen AM, Lalka SG, et al. Three-dimensional gadolinium-enhanced MR angiography for aortoiliac inflow assessment plus renal artery screening in a single breath-hold. Radiology 1996;98:725-32.

[17] Earls JP, Patel NH, Smith PA, DeSena S, Meissner MH. Gadolinium-enhanced three-dimensional MR angiography of the aorta and peripheral arteries: Evaluation of a multistation examination using two gadopentetate dimeglumine infusions. AJR Am J Roentgenol 1998;171:599-604.

[18] Dubois J, Lethimonnier F, Vaufrey F, Robert P, Le Bihan D. On the use of TrueFISP sequence for the detection of dysprosium-DOTA in angiography (in French). Proceedings of the 10th Annual Meeting of GRAMM. Angers, France; 2003 [VD1].

[19] Dubois J, Lethimonnier F, Vaufrey F, Robert P, Le Bihan D. Frequency-shift based detection of contrast agents using SSFP: Potential for MRA. Proceedings of the 11th Annual Meeting of ISMRM. Toronto, Canada; 2003. p. 321.

[20] Haacke EM, Brown RW, Thomson MR, Venkatesan R, editors. Magnetic resonance imaging: Physical principles and sequence design. New York: Wiley-Liss; 1999. p. 451-512.

[21] Haacke EM, Wielopolski PA, Tkach JA, Modic MT. Steady-state free precession imaging in the presence of motion: Application for improved visualization of the cerebrospinal fluid. Radiology 1990; 175:545-52.

[22] Zur Y, Stokar S, Bendel P. An analysis of fast imaging sequences with steady-state transverse magnetization refocusing. Magn Reson Med 1988;6:175-93.

[23] Gyngell ML. The steady-state signals in short-repetition-time sequences. J Magn Reson 1989;81:474-83.

[24] Duerk JL, Lewin JS, Wendt M, Petersilge C. Remember TrueFISP? A high SNR, near 1-second imaging method for $\mathrm{T}_{2}$-like contrast in interventional MRI at 0.2T. J Magn Reson Imaging 1998;8:203-8.

[25] Le Roux P. Simplified model and stabilization of SSFP sequences. J Magn Reson 2003;163:23-37.

[26] Fossheim S, Johansson C, Fahlvik AK, Grace D, Klaveness J. Lanthanide-based susceptibility contrast agents: Assessment of the magnetic properties. Magn Reson Med 1996;35:201-6.

[27] Villringer A, Rosen BR, Belliveau JW, Ackerman JL, Lauffer RB, Buxton RB, et al. Dynamic imaging with lanthanide chelates in normal brain: Contrast due to magnetic susceptibility effects. Magn Reson Med 1988;6:164-74.
[28] Chu SCK, Xu Y, Balschi JA, Springer Jr CS. Bulk magnetic susceptibility shifts in NMR studies of compartmentalized samples: Use of paramagnetic reagents. Magn Reson Med 1990;13:239-62.

[29] Springer Jr CS, Xu Y. Aspects of bulk magnetic susceptibility in in vivo MRI and MRS. In: Rinck PA, Muller RN, editors. New developments in contrast agent research, European Magnetic Resonance Forum. Blonay, Switzerland; 1991. p. 13-25.

[30] Haacke EM, Brown RW, Thomson MR, Venkatesan R, editors. Magnetic resonance imaging: Physical principles and sequence design. New York: Wiley-Liss; 1999. p. 741-79.

[31] Springer Jr CS. Physicochemical principles influencing magnetopharmaceuticals. In: Gillies RJ, editor. NMR in physiology and biomedicine. San Diego: Academic Press; 1994. p. 76-99.

[32] Fossheim S, Saebo KB, Fahlvik AK, Rongved P, Klaveness J. Low molecular weight lanthanide contrast agents: In vitro studies of mechanisms of action. J Magn Reson Imaging 1997;7:251-7.

[33] Weisskoff RM, Kiihne S. MRI susceptometry: Image-based measurement of absolute susceptibility of MR contrast agents and human blood. Magn Reson Med 1992;24:375-83.

[34] Corot C, Violas X, Robert P, Port M. Pharmacokinetics of three gadolinium chelates with different molecular sizes shortly after intravenous injection in rabbits: Relevance to MR angiography. Invest Radiol 2000;35(4):213-8.

[35] Dupas B, Bach-Gansmo T, Meflah K, Muller RN. Synergistic effects of relaxation and susceptibility in differentiation between compartmentalized and noncompartmentalized tissues. Invest Radiol 1998;33:268-72.

[36] Patz S. Some factors that influence the steady state in steady-state free precession. Magn Reson Imaging 1988;6:405-13.

[37] Scheffler K. On the flow sensitivity of balanced SSFP imaging. Proceedings of the 14th Annual International Workshop on MR Angiography. Essen, Germany; 2002. p. 121.

[38] Miraux S, Thiaudiere E, Canioni P, Franconi J. Chemical-shift angiography (CSA) with dysprosium-DOTA. Proceedings of the 20th Annual Meeting of ESMRMB. Rotterdam, Netherlands; 2003. p. 59.

[39] Freeman R, Hill HDW. Phase and intensity anomalies in Fourier transform NMR. J Magn Reson 1971;4:366-83.

[40] Hargreaves BA, Vasanawala SS, Nayak KS, Hu BS, Nishimura DG. Fat-suppressed steady-state free precession imaging using phase detection. Magn Reson Imaging 2003;50:210-3. 